\title{
Key Technologies for Collaborative Robots in Front-end Retail Automation
}

\author{
Gustavo Alfonso Garcia Ricardez*, Jun Takamatsu* and Tsukasa Ogasawara*
}

*Nara Institute of Science and Technology

\section{Introduction}

In recent years, robotic applications have been evolving from working for humans to working with humans. This evolution is driven by the need of merging the human and robot skills, a mainly economic incentive for societal continuance. Robots can supply the increasing demand of workforce[1], especially patent in aging societies, but even state-of-the-art robotic technology can come short in terms of robustness and efficiency, dexterity and interaction, and decision-making. Among other service robotics applications, one that has been the focus of attention is front-end retail automation, i.e., the automation of restocking, straightening, cleaning and customer interaction. This is patent in the Future Convenience Store Challenge (FCSC) of the World Robot Challenge (WRC) [2], which gathers the community to innovate the collaboration between staff and robots to efficiently operate an establishment, as well as between robots and customers to provide a better shopping experience. In this paper, we discuss the technologies we have identify as key to drive a paradigm shift in the humanrobot collaboration in front-end retail scenarios, necessary to deploy safe, efficient and intelligent robots in our daily life.

\section{Challenges}

Through our participation in the FCSC 2018 and in preparation for the FCSC 2020, we have identified key technologies to achieve a harmonic synergy between humans and robots in a convenience store scenario.

Human safety: Human-Robot Interaction (HRI) in a retail establishment often occurs in close proximity (less than $1.5[\mathrm{~m}]$ ) due to the optimized space for product display. Moreover, the robot needs to operate with constant human interruptions and surrounded by both

\footnotetext{
原稿受付 2019 年 10 月 1 日

キーワード : Collaborative Robots, Retail Automation, Human Safety, Human-robot Interaction, Robotics Competitions

*テ630-0192 生駒市高山町 8916-5

*Ikoma-shi, Nara
}

customers and staff. Though human safety technologies have been developed, for example, we have previously proposed reactive strategies to modify the robot speed to reduce the impact of the trade-off between human safety and efficiency that affects the robot's performance [3] [4], a smart monitoring of the human state is still needed. This is because cameras fixed on the environment (e.g., surveillance cameras) are prone to occlusions and cannot provide all the necessary view points to ensure human safety, in particular for regions between the robot manipulators and the human body where the risk of injury is higher.

Human comfort: Once the physical safety is ensured, technologies to improve the human perception of safety as well as the comfort when operating or working with robots are needed. For example, we have investigated the effects of the food delivery location of an eating assistive device (see Fig. 1), whose placement in front of the user has been conventionally decided in a roughly manner, and may discourage users to adopt such technologies [5]. Besides the perception of safety,

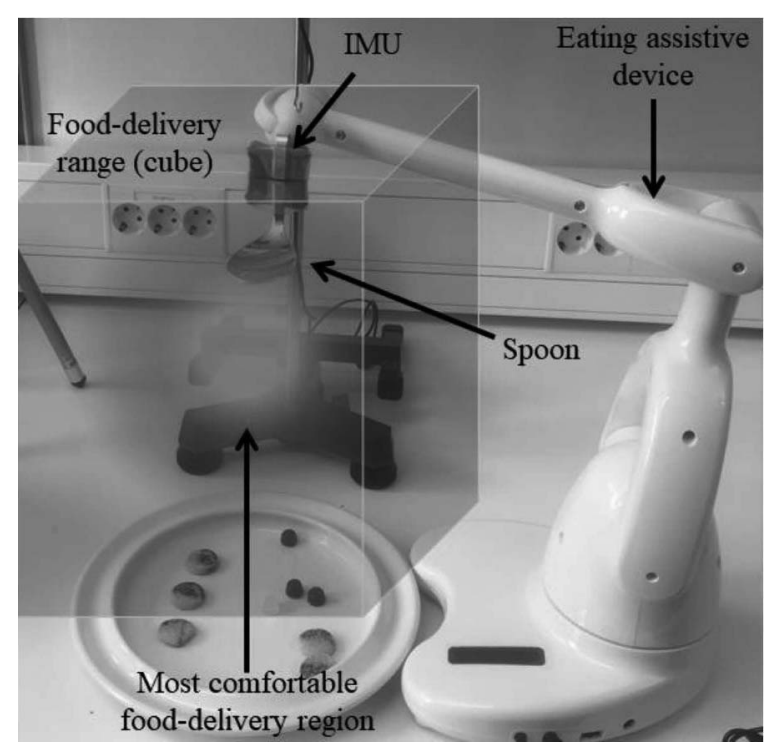

Fig. 1 Comfort estimation of an eating assistive device using IMU sensors to indirectly estimate the interaction force. Regions with lower interaction forces are more comfortable 
the negative effects of getting used to pseudo-safe technologies should also be studied because humans naturally disassociate danger from estimable phenomena.

Efficient HRI: The collaboration success goes beyond the individual efficiency of either humans or robots but rather of both. Conventionally, the robot stops when safety constraints are met, even when using robotefficient methods such as AVM[3], but the robot may end up in the way of the human. The robot needs to a) remove itself from the way by withdrawing its end effector and assuming a previously asserted safe configuration [6], which may increase human efficiency, or b) identify the opportunity of collaboration to avoid withdrawing and perform the part of the human task that is compromised. For example, when the human needs to reach an item in a shelf but the robot arm is on the way, instead of standing still or withdrawing, the robot grasps the item and hands it out to the human. This is expected to increase the efficiency of both by reducing the time the robot pauses its task and the time the human needs to wait for the robot to move away.

Safety-efficiency-comfort trade-off: The collaborative behavior of the robot should be tuned to the task, the human collaborator, the environment, and so on, and it is bound to safety, comfort and efficiency requirements. As one criterion gets stricter or relaxed, the others are consequently affected. For example, the collaboration with staff requires less comfort but maximum efficiency, while the collaboration with customers requires maximum comfort and efficiency becomes sacrificable. While all behaviors are expected to be safe, the risk of harm needs also to be managed and, as the other criteria do, safety should be tuned to the collaboration requirements. This example is depicted in Fig. 2.

Human psychological alleviation: Beyond the safety and comfort that robots operation could achieve, we foresee the need of robotic therapy based on physical contact with the specific purpose of improving the mental state of customers. These applications are well exemplified by Paro [7] and touch care [8], a mascotlike robot that provides the users a channel to transmit caring feelings, and a therapeutic service that robots provide by caressing the humans in a comforting fashion (see Fig. 3), respectively.

Communication: Human-like communication requires to develop advanced behaviors to refine the existing communication methods. For example, we can verbally communicate with robots but natural communication usually involves interruptions [9]. When we are talking to a robot, we expect it to establish eye contact [10] but we also need the robot to avoid continu-

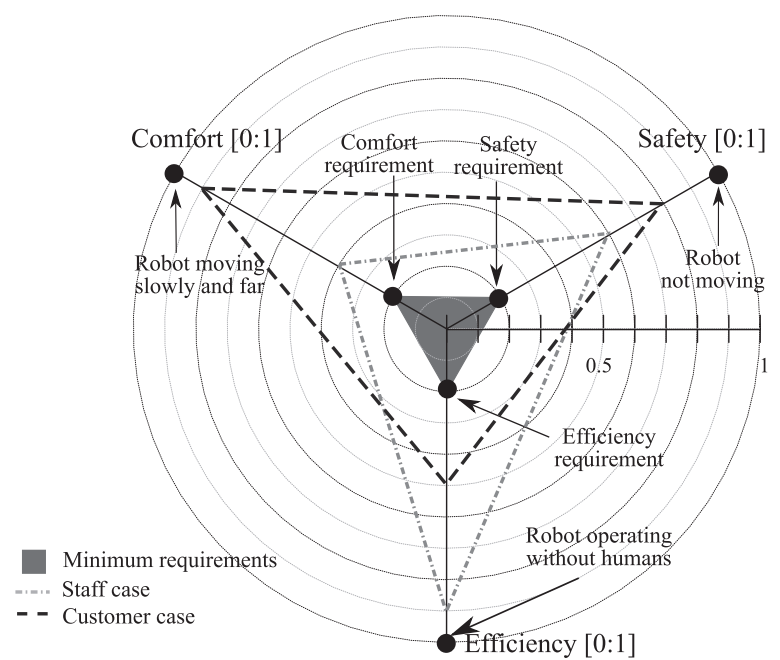

Fig. 2 Comparison of different collaboration settings in terms of human safety, efficiency and comfort in HRI. When collaborating with staff, the robot can reduce the comfort and safety in order to gain efficiency. When collaborating with customers, efficiency can be sacrificed in order to maximize comfort and safety

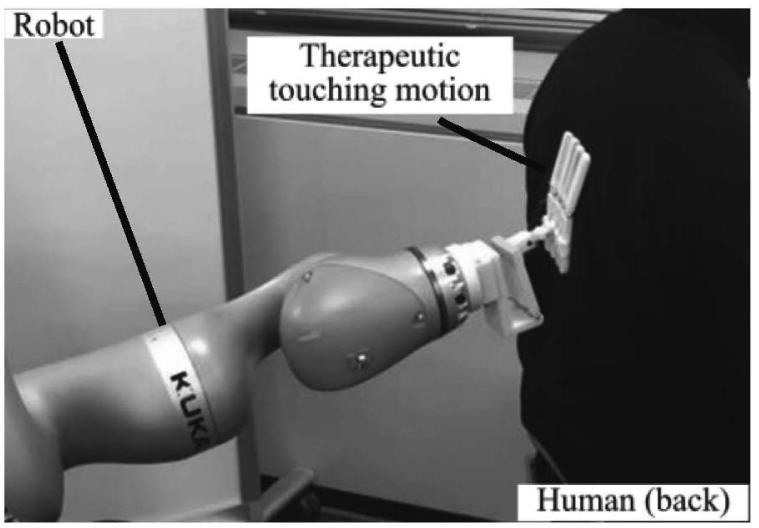

Fig. 3 Therapeutic touching motion performed by a robot to psychologically alleviate a human, for example, from stress

ously staring and break the eye contact in the proper way and timing to give the sensation of politeness. Finally, while gesture-based communication has been mainly focused on the recognition of human gestures by the robots, we need the robots to gesture back in ways that are not only easy to understand but also elegant or aesthetically pleasant [11] (see Fig. 4).

Adaptive collaboration: In a retail scenario, we need to adapt the robot behavior to different types of collaborative tasks:

- Simultaneous tasks: both human and robot are manipulating the same object at the same time, e.g., moving a shelf together. The robot needs to switch between master-slave and slave-master modes.

- Intermittent tasks: human and robot are working toward the same goal but each has their set of subtasks. These tasks can be independent, e.g., restocking differ- 


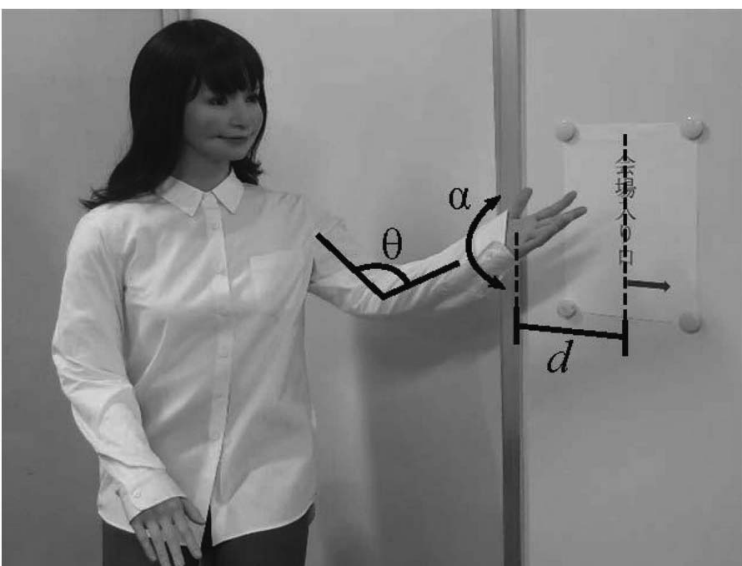

Fig. 4 Elegant motions implemented on an Actroid robot. Motions with different perceptions can be generated by changing parameters such as the wrist rotation $\alpha$, the elbow angle $\theta$, and the distance $d$ to the pointed object

ent bins in a shelf, or dependent, e.g., filling the same bin or filling a box with items. The robot needs to find the proper timing to interlace its subtasks.

- Supervised tasks: The human supervises the robot execution of a task to intervene if necessary. The robot needs to broadcast (e.g., using mixed reality [12]) its state and plans so a human can be aware of the proper or faulty operation while doing other tasks.

- Hanging tasks: The completion of the human subtask depends on the completion of the robot subtask, or vice versa, e.g., holding a bag for the other to put items. The robot needs to identify when the objective of the task is achieved to finish the collaboration, or otherwise wait until completion.

While these technologies seem promising even separately, there is the need to integrate them into a versatile and reliable framework. Ideally, this effort should be community-driven and produce an open source framework, whose contributions can push the sooner inclusions of collaborative robots in retail environments.

\section{Research opportunities}

Recently, the number of opportunities to research, develop and test robotic technologies are increasing by the creation of international robotics competitions such as the FCSC, the Partner Robot Challenge [13] at the WRC, and RoboCup@Home[14], among others. As technological advances allow, requirements for collaborative tasks have been also included. For example, the 2020 edition of the FCSC explicitly requires the robot to modify its behavior when a customer wants to take an item from the shelf while the robot restocks it, or enter the restroom while the robot is cleaning. Furthermore, research grants have started to require higher technol- ogy readiness levels in order to enforce the last mile of the research and development race, where a proof of concept is not sufficient and system prototype demonstrations in operational environments are demanded.

\section{Conclusion}

The key technologies for collaborative robots for front-end retail automation include safety, efficiency and comfort, as well as a more effective exploitation of their trade-off. Moreover, psychological alleviation, a richer verbal and non-verbal communication, and adaptive behaviors for different types of tasks are also needed. Finally, we suggest that a good opportunity to take these technologies to higher levels of technology readiness is by participating in international robotics competitions.

\section{References}

[1] N. Kishida and H. Nishiura: "Demographic supply-demand imbalance in industrial structure in the super-aged nation Japan," Theoretical Biology and Medical Modelling, vol.15, no.1, p.19, 2018.

[2] K. Wada: "New robot technology challenge for convenience store," Proc. of the 2017 IEEE/SICE International Symposium on System Integration (SII 2017), pp.1086-1091, 2017.

[3] G.A. Garcia Ricardez, A. Yamaguchi, J. Takamatsu and T. Ogasawara: "Asymmetric Velocity Moderation for human-safe robot control," Advanced Robotics, vol.29, no.17, pp.1111$1125,2015$.

[4] G.A. Garcia Ricardez, A. Yamaguchi, J. Takamatsu and T. Ogasawara: "Human safety index based on impact severity and human behavior estimation," Mechatronics and Robotics Engineering for Advanced and Intelligent Manufacturing, pp.177190, Springer, 2016.

[5] G.A. Garcia Ricardez, J. Solis Alfaro, J. Takamatsu and T. Ogasawara: "Quantitative comfort evaluation of eating assistive devices based on interaction forces estimation using an accelerometer," Proc. of the 27th IEEE International Symposium on Robot and Human Interactive Communication (RO-MAN 2018), pp.909-914, 2018

[6] G.A. Garcia Ricardez, A. Yamaguchi, J. Takamatsu and T. Ogasawara: "Withdrawal strategy for human safety based on a virtual force model," Proc. of the 2013 IEEE/RSJ International Conference on Intelligent Robots and Systems (IROS 2013), pp.1119-1124, 2013.

[ 7 ] K. Wada, Y. Ikeda, K. Inoue and R. Uehara: "Development and preliminary evaluation of a caregiver's manual for robot therapy using the therapeutic seal robot Paro," Proc. of the 19th International Symposium in Robot and Human Interactive Communication (RO-MAN 2010), pp.533-538, 2010.

[8] K. Toyoshima, D. Ming, J. Takamatsu and T. Ogasawara: "What is required for a robot to gently stroke a human using its hand," Proc. of the ICRA 2018 Workshop on Elderly Care Robotics - Technology and Ethics, 2018.

[ 9 ] Y. Kondo, K. Takemura, J. Takamatsu and T. Ogasawara: "A gesture-centric android system for multi-party human-robot interaction," Journal of Human-Robot Interaction, vol.2, no.1, pp.133-151, 2013.

[10] A. Yuguchi, G.A. Garcia Ricardez, M. Ding, J. Takamatsu and T. Ogasawara: "Gaze calibration for human-android eye contact using a single camera," Proc. of the 2017 IEEE International Conference on Robotics and Biomimetics (ROBIO 2017), pp.883-888, 2017.

[11] M. Ikawa, E. Ueda, A. Yuguchi, G.A. Garcia Ricardez, M. Ding, J. Takamatsu and T. Ogasawara: "Quantification of elegant 
motions for receptionist android robot," Proc. of the International Conference on Digital Human Modeling and Applications in Health, Safety, Ergonomics and Risk Management, pp.435$446,2017$.

[12] H. Nakamura, L. El Hafi, Y. Hagiwara and T. Taniguchi: "Calibration system using semantic-ICP for visualization of robot spatial perception through Mixed Reality," Proc. of the 2019 Annual Conference of the Japanese Society for Artificial Intel- ligence (JSAI 2019), pp.1-4, 2019

[13] H. Okada, T. Inamura and K. Wada: "What competitions were conducted in the service categories of the World Robot Summit?," Advanced Robotics, vol.33, no.17, pp.1-11, 2019.

[14] T. Wisspeintner, T. Van Der Zant, L. Iocchi and S. Schiffer: "RoboCup@Home: Scientific competition and benchmarking for domestic service robots," Interaction Studies, vol.10, no.3, pp.392-426, 2009.

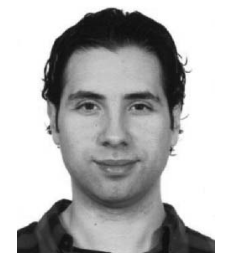

Gustavo Alfonso Garcia Ricardez

Gustavo Alfonso Garcia Ricardez received his M.E. and Ph.D. degrees from the Nara Institute of Science and Technology, Japan, in 2013 and 2016, respectively. $\mathrm{He}$ is currently an Assistant Professor in the Robotics Laboratory of the Division of Information Science at the Nara Institute of Science and Technology. His research interests include human-safe, efficient robot control, human-robot interaction, manipulation and task planning, and he leads numerous research projects and teams in international robotics competitions.

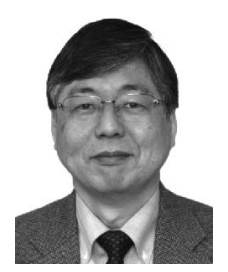

\section{Tsukasa Ogasawara}

Tsukasa Ogasawara received his Ph.D. degree from the University of Tokyo, Japan, in 1983. From 1983 to 1998, he was with the Electrotechnical Laboratory, Ministry of International Trade and Industry, Japan. From 1993 to 1994, he was with the Institute for Real-Time Computer Systems and Robotics, University of Karlsruhe, Germany, as a Humboldt Research Fellow. He joined the Nara Institute of Science and Technology, Nara, Japan, in 1998, where he is currently a Professor in the Division of Information Science. He is also Vice President of the Nara Institute of Science and Technology and Dean of the Graduate School of Science and Technology. His research interests include human-robot interaction, dexterous manipulation, human modeling and bio-inspired robotics.

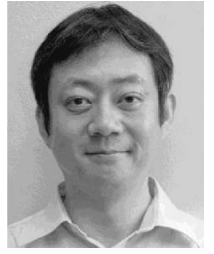

\section{Jun Takamatsu}

Jun Takamatsu received his Ph.D. degree in Computer Science from the University of Tokyo, Japan, in 2004. From 2004 to 2008, he was with the Institute of Industrial Science, University of Tokyo. In 2007, he was with Microsoft Research Asia, as visiting researcher. In 2008, he joined the Nara Institute of Science and Technology, Japan, as Associate Professor. His research interests include task and motion planning, learning from observation, feasible motion analysis, 3D-shape modeling and analysis, and physics-based vision. 